\title{
Compliance with Pharmacotherapy and Direct Healthcare Costs in Patients with Parkinson's Disease: A Retrospective Claims Database Analysis
}

\author{
Florent F. Richy • Guilhem Pietri • Kimberly A. Moran • \\ Emmanuelle Senior • Lydia E. Makaroff
}

Published online: 7 May 2013

(c) The Author(s) 2013. This article is published with open access at Springerlink.com

\begin{abstract}
Background Parkinson's disease (PD) is a progressive neurological disorder for which, at present, there is no cure. Current therapy is largely based on the use of dopamine agonists and dopamine replacement therapy, designed to control the signs and symptoms of the disease. The majority of current treatments are administered in tablet form and can involve multiple daily doses, which may contribute to sub-optimal compliance. Previous studies with small groups of patients suggest that non-compliance with treatment can result in poor response to therapy and may ultimately increase direct and indirect healthcare costs.

Objective To determine the extent of non-compliance within the general PD population in the USA as well as the patient characteristics and healthcare costs associated with compliance and non-compliance.

Methods A retrospective analysis from a managed care perspective was conducted using data from the USA PharMetrics patient-centric claims database. PharMetrics claims data were complete from 31 December 2005 to 31 December 2009. Patients were included if they had at least two diagnoses for PD between 31 December 2005 and 31
\end{abstract}

F. F. Richy · E. Senior · L. E. Makaroff ( $\square$ )

UCB Pharma, Allée de la Recherche 60, 1070 Brussels, Belgium

e-mail: Lydia.Makaroff@ucb.com

F. F. Richy

Liège University, Liège, Belgium

G. Pietri

HERON Evidence Development Ltd, Luton, UK

K. A. Moran

UCB Pharma, Smyrna, USA
December 2008, were older than 18 years of age, were continuously enrolled for at least 12 months after the date of the most recent PD diagnosis, and had no missing or invalid data. The follow-up period was the most recent 12-month block of continuous enrollment that occurred between 2006 and 2009. Patients were required to have at least one PD-related prescription within the follow-up period. The medication possession ratio (MPR) was used to categorise patients as compliant or non-compliant. Direct all-cause annual healthcare costs for patients with PD were estimated for each patient, and regression analyses were conducted to determine predictors for non-compliance.

Results A total of 15,846 patients were included, of whom $46 \%$ were considered to be non-compliant with their prescribed medication (MPR <0.8). Predictors of non-compliance included prescription of a medication administered in multiple daily doses $(p<0.0001)$, a period of $<2$ years since the initial PD diagnosis $(p=0.0002)$, a diagnosis of gastrointestinal disorder $(p<0.0001)$, and a diagnosis of depression $(p<0.0001)$. Non-compliance was also found to be related to age, with a lower odds of non-compliance in patients aged $41-80$ years than in patients aged $\geq 81$ years $(p<0.05)$. Although total drug mean costs were higher for compliant patients than non-compliant patients (driven mainly by the cost of PD-related medications), the mean costs associated with emergency room and inpatient visits were higher for patients non-compliant with their prescribed medication. Overall, the total all-cause annual healthcare mean cost was lower for compliant $(\$ 77,499)$ than for non-compliant patients $(\$ 84,949 ; p<0.0001)$.

Conclusion Non-compliance is prevalent within the general USA PD population and is associated with a recent PD diagnosis, certain comorbidities, and multiple daily treatment dosing. Non-compliance may increase the burden on the healthcare system because of greater resource usage 
compared with the compliant population. Treatments that require fewer daily doses may have the potential to improve compliance, which in turn could reduce the economic burden associated with PD.

\section{Key Points for Decision Makers}

- Almost half of all patients with Parkinson's disease in this study were non-compliant with their prescribed medication, with non-compliance associated with higher resource utilisation and greater direct all-cause healthcare mean costs.

- Predictors of non-compliance in the Parkinson's disease population were: older age, multiple daily dosing, more recent PD diagnosis, and the presence of depression or gastrointestinal disorder.

\section{Introduction}

Parkinson's disease (PD) is a progressive neurological disorder caused by the degeneration of dopaminergic neurons in the substantia nigra, an area of the midbrain that plays a crucial role in voluntary motor control. It is the second most common neurological disease worldwide following Alzheimer's disease and is the most widespread form of Parkinsonism. PD should be suspected in patients presenting with bradykinesia, rigidity, tremors, and/or postural instability [1-3]. PD is an age-related disorder with a mean onset of 57 years [4], and the prevalence increasing with age; in the USA, PD is diagnosed in approximately 128 cases in every 100,000 individuals aged 50-54 years, with this figure increasing to approximately 958 cases in every 100,000 individuals aged 75-79 years [5]. An estimated 5 million individuals worldwide have PD, with 1 million patients diagnosed within the USA [3].

At present there is no cure for PD; modern therapy is largely based on a strategy that is designed to control the signs and symptoms associated with PD using dopamine replacement and dopamine agonists. Several drug classes are used for the treatment of patients with PD, with current regimens centred chiefly on the dopamine precursor levodopa [6]. Though initially effective, within 5 years of initiating levodopa therapy, up to $80 \%$ of patients will experience substantial side effects such as the development of fluctuations in motor performance, dyskinesias, and neuropsychiatric complications [7]. Thus, other treatments such as dopamine agonists, amantadine, and monoamine oxidase (MAO-B) inhibitors may be used as first-line monotherapy to initially control PD.

Current EU and North American guidelines recommend initial treatment with a dopamine agonist, an MAO-B inhibitor, or levodopa in early PD [2, 8-11]; the choice relies on patient characteristics (including age of onset and comorbidities) and the degree of disability. This levodopasparing strategy can reduce the risk of motor complications at the early stages of treatment $[3,12]$. In advanced $\mathrm{PD}$, levodopa continues to be the most effective therapy for the motor symptoms of the disease [3]. However, the resulting pulsatile dopaminergic stimulation may be associated with motor fluctuations and complications, which has been partially supported by historical evolution of the levodopa dose over decades and by clinical trial data [13].

Many PD-related treatments are administered in oral form and can involve multiple oral agents. These agents generally have different administration/dosing schedules, with the resulting regimen complexity making it difficult for patients to remain compliant to treatment. Indeed, initial reports have indicated that there may be widespread irregularity between patients in their compliance with PD treatment $[14,15]$, with one study estimating that $46 \%$ of patients taking orally delivered medications are non-compliant [16]. Compliance can be defined as the extent to which a patient's actual drug timing, administration, and dose corresponds to that which is prescribed for them; in clinical trials, compliance is usually measured by a tablet count [17]. In general, non-compliance is defined as $<80 \%$ of prescribed tablet intake using the medication possession ratio (MPR), a formula used to determine patient compliance with their specific treatment regimen [18]. The MPR is calculated as the sum of the non-overlapping total days supplied across all PD-related prescriptions in a given period, divided by the number of days between the first and last prescription plus the days supplied at the last prescription. The MPR, therefore, gives a numerical measure of how many non-overlapping days of therapy were available to the patient within the timeframe measured and compares this to the total number of days from the first prescription to the end of the last prescription.

Approximately $50 \%$ of patients with chronic diseases living in developed countries do not follow treatment recommendations $[19,20]$. For patients with PD, there is growing evidence to suggest that sub-optimal compliance is a contributing factor to the variable response to dopamine replacement therapy [21]. For example, a multicentre European study found that non-compliance with PD treatment was significantly associated with poor motor scores [on the unified PD rating scale (UPDRS)], more daily "off" time (from UPDRS 4), and worse mobility [within the PD quality of life score (PDQ39)] compared with patients who demonstrated satisfactory compliance [21, 22]. Further, non-compliance has been found to be associated with higher rates of depression and reduced quality of life [14]. 
This variable response to treatment may have an economic impact. In North America, the direct medical cost of treating patients with PD is high: in the USA alone it is estimated to be $\$ 6.7$ billion (2002 US dollars) per year in total [13]. The drivers identified for this economic burden included outpatient service costs $(24 \%$ of the total direct cost), hospitalisation costs $(15 \%$ of the total direct cost), and the cost of prescription drugs (14\% of the total direct cost) [13]. Similarly, a recent observational study estimated that the annual cost of PD in the USA was approximately $\$ 10.78$ billion (2007 US dollars), with direct costs accounting for $\$ 6.22$ billion and indirect costs accounting for $\$ 4.56$ billion per year [23]. The variable response to dopamine replacement therapy observed in patients with PD is likely to affect the direct healthcare costs incurred, with higher costs expected in PD populations for whom the medication is less effective, either through lack of efficacy or noncompliance.

Therefore, the aim of the study was to determine the distribution and extent of non-compliance within the insured USA PD population, characteristics of patients who are compliant versus those who are non-compliant, predictors of non-compliance, and healthcare costs associated with non-compliance. It was hypothesised that non-compliance would be observed in a substantial proportion of patients with PD, similar to that observed previously, and that this non-compliance would result in a higher yearly healthcare cost than that associated with patients compliant with treatment.

\section{Methods}

\subsection{Database}

This retrospective analysis used a datacut derived from the PharMetrics patient-centric claims database that comprises longitudinal de-identified information from enrolment files as well as facility, professional service, and outpatient pharmacy claims from private healthcare benefit plans covering over 55 million patients enrolled in more than 90 health plans across the USA. The PharMetrics integrated database includes medical and pharmacy claims with International Classification of Diseases, Ninth Revision, Clinical Modification (ICD-9-CM) codes; all claims for a given patient are linked using a unique encrypted identifier. The data set is compliant with the Health Insurance Portability and Accountability Act (HIPAA). The database is geographically diverse and is considered to be representative of the commercially insured population in the USA
[24]. The datacut used in this study included all patients having a claim for an ICD-9 diagnosis code of PD (332.XX) during the period 1 January 2000 to 31 December $2009(N=95,076)$.

\subsection{Study Design and Patient Selection}

This study was conducted from a managed care perspective. The PharMetrics datacut used for this study contained data complete from 31 December 2005 to 31 December 2009. Patients were included in the sample used for the analysis if they had at least two diagnoses for PD (ICD-9 332) between 31 December 2005 and 31 December 2008, if they were older than 18 years of age at the time of the most recent $\mathrm{PD}$ diagnosis, were continuously enrolled for at least 12 months after the date of the most recent PD diagnosis, and had no missing or invalid data. The follow-up period occurred between 2006 and 2009, and costs were not adjusted for inflation. Two PD diagnoses were mandated to increase the specificity of this inclusion criterion. All health plans included within the PharMetrics database were included. Once two PD diagnoses had been established for each patient, the most recent 12 months of continuous enrolment was defined as the follow-up period, and the first date of this period was classed as the index date (Fig. 1). Patients were required to have at least one PD-related prescription within the follow-up period. Thus, the index date was not necessarily the date of service of either a PD medication fill or a PD diagnosis. The "number of medications" was defined as the number of PD-related pharmaceutical claims for distinct non-proprietary compounds during the follow-up period. The "number of prescriptions" was defined as the total number of PD-related pharmaceutical claims submitted during the follow-up period. The "number of days supplied" field on each paid pharmacy claim was used to calculate therapy days. If the number of days supplied was invalid (i.e. missing or equal to zero), wherever possible the number was imputed using the last observation carried forward (LOCF) method; patients were excluded if the number of days supplied was invalid and could not be imputed in this manner. Patients with 50 or more PD-related tablets a day were also excluded to reduce the influence of outliers.

\subsection{Patient Assessment and Non-Compliance}

Non-compliance was defined as patients being without a claim for PD-related medication for $>20 \%$ of the 12-month follow-up period and compliance defined as an overall MPR $\geq 80 \%$ for all PD-related drugs prescribed [16]. The MPR was calculated using the formula: 

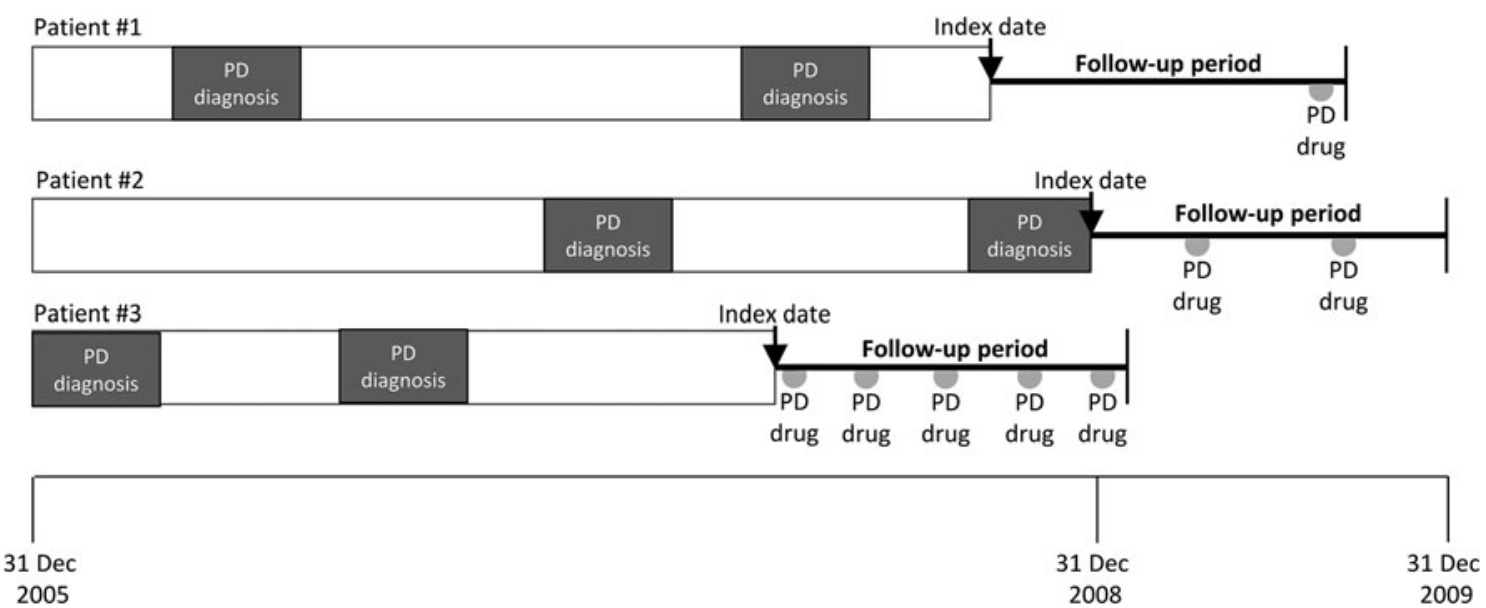

Fig. 1 Study design indicating index date and follow-up period. All patients were required to have at least one PD-related prescription during the 12-month follow-up period

Total non - overlapping days supplied of all PD - related prescriptions during follow - up period Number of days between the first and last prescription plus the days supplied of the last prescription

PD-related medication was defined as anticholinergics (e.g., benztropine, biperiden, ethopropazine, procyclidine, trihexyphenidyl; GPI code starting with 7310), COMT inhibitors (tolcapone, entacapone; 7315), amantadine (7320001), dopamine agonists [e.g., bromocriptine, cabergoline, pergolide, pramipexole (immediate and extended release), ropinirole (immediate and extended release); 7320002, 7320005, 73203], levodopa [including levodopa/ carbidopa and levodopa/carbidopa/entacapone] (7320004, 73209), and MAO-B inhibitors (e.g., rasagiline, selegiline; 733). These medications were included regardless of their form or dosage (e.g., once daily/extended-release dopamine agonists were included alongside dopamine agonists requiring multiple daily dosing). All other medications received were classed as non-PD-related drugs.

Age at index date was calculated using the derived year of birth. Gender was equivalent to the sex listed on the enrolment record. Region was assigned as East, Northwest, South, or West, as defined by the US Census Bureau. The regimen was defined according to the generic ingredients prescribed during the follow-up period. The number of pills per day was calculated as the sum of the quantity dispensed divided by the number of days supplied. If the days supplied field was missing, the field was imputed from the package insert for that medication. Medications were classified as requiring multiple daily treatment doses according to instructions on their package inserts. Time since PD diagnosis was defined as the number of days between the index date and the patient's first PD diagnosis in the database.

Depression and gastrointestinal disorders (GIDs) have previously been identified as important comorbidities in PD [25, 27] and were thus defined as such in this analysis. Patients were classified as having depression if they had both a diagnosis for a depressive or dysthymic disorder and a prescription for an antidepressant (GPI code began with 58; ICD-9 codes 296.3, 311, 300.4, 296.2). A diagnosis of depression could occur at any time before or during the follow-up period. Patients were classified as having a GID if they were diagnosed with oesophageal disease, an ulcer, gastritis, stomach disorders, mucositis, intestinal obstruction, digestive disorders, peritoneum disorders, intestinal disorders, or dysphagia (ICD-9 codes 787.2, 530-538, 560, 564, 568, 569) (see Makaroff 2011). Similarly, a diagnosis of GID could occur at any time before or during the followup period.

\subsection{Cost Estimation}

Total all-cause annual healthcare costs were calculated and stratified by medication (PD-related medication and nonPD medication), emergency room visits, laboratory investigations, non-laboratory services, physician visits, and inpatient and uncategorised costs. These costs were 
estimated for each patient from the PharMetrics database using the variable "ALLOWED", which reflects the amount the plan allows for a particular service. It is typically the paid amount plus any member liability (e.g., copayment, deductible, and coinsurance). Outpatient costs were the sum of emergency room, laboratory, non-laboratory ancillary service, and physician visit costs. Inpatient costs were calculated from non-emergency room hospitalisation costs. Drug costs were the sum of PD-related drug and other drug costs. Total costs were the sum of these outpatient and drug costs, plus inpatient and uncategorised costs.

\subsection{Statistical Methods}

For continuous variables (e.g., mean age, total cost), a twosample $t$ test was conducted to compare the mean outcome values between compliant (MPR $>0.8$ ) and non-compliant (MPR $\leq 0.8)$ patients. Where the continuous samples being compared were found to have equal variance, a pooled analysis was used (default analysis); a Satterthwaite analysis was used if unequal variances were observed. For categorical variables (e.g., age categories, number of pills per day), the Pearson's chi-square test was used to compare the distribution of patients across the compliant and noncompliant cohorts. Statistical significance was assessed at $p \leq 0.05$ (alpha value). Age was treated as a discrete variable since the differences between each year could not be assumed to be equivalent. The total number of tablets was collapsed into a dichotomous variable in which both categories contained approximately the same number of patients (i.e. $<10$ tablets per day, $>10$ tablets per day) because this variable was not normally distributed.

A multiple logistic regression model was built to determine the predictors that impact upon compliance. The dependent variable of the model was compliance (MPR $<80$ vs. $\geq 80 \%$ ). Within each covariate, a reference group was assigned to define a base case to which other groups could be compared. The choice of reference group was arbitrary. A stepwise (backwards) selection of the covariates was conducted to assess the statistical significance of each covariate as a predictor of non-compliance and to remove covariates that were considered non-statistically significant if their $p$ value from the type 3 analysis was $>0.05$ (alpha value). For a variable with multiple categories such as age or dosing regimen, all categories were required to be non-significant predictors for the variable to be removed from the model. Covariates potentially influencing the probability of non-compliance were patient demographics (age and gender), drug characteristics (number of tablets per day and prescription for daily tablets), and medical characteristics (time since PD diagnosis, presence of GID, and diagnosis of depression).

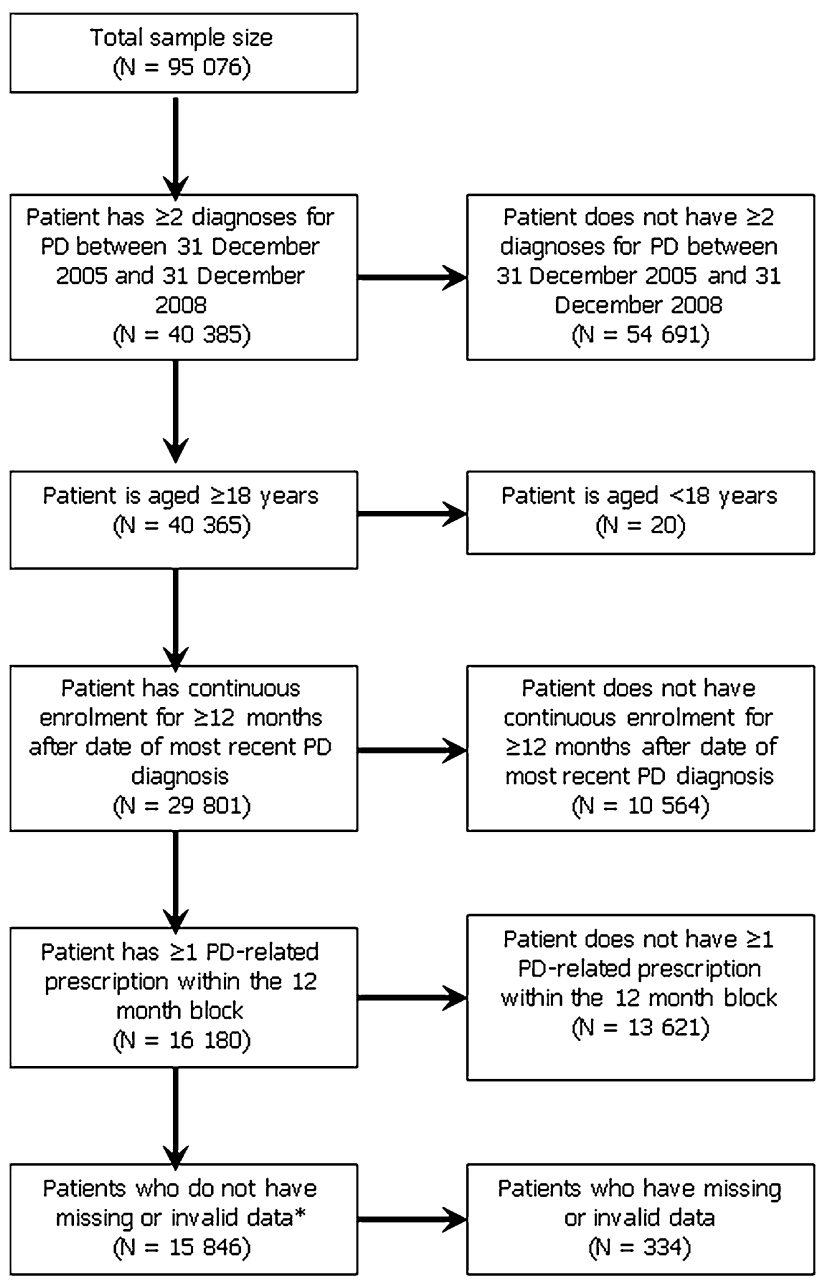

Fig. 2 Flow chart indicating patient selection from the PharMetrics ${ }^{\circledR}$ database. $P D$ Parkinson's disease. * Patients were deleted because of missing or zero values for quantity after last observation carried forward (LOCF) was used. Patients were also excluded if they had PD prescriptions for 50 or more tablets a day

All analyses were conducted using the $\mathrm{SAS}^{\circledR}$ statistical package (version 9.2; SAS Institute Inc., NC, USA), including study design, patient selection, and data analysis.

Due to the observational retrospective design of this study, no causal links could be established among compliance, cost, and comorbidities

\section{Results}

The flow of patients through the analysis is detailed in Fig. 2. A total of 15,846 patients with PD were identified from the PharMetrics database, of whom $60 \%$ were male, $35 \%$ had a diagnosis of depression, and $65 \%$ had a diagnosis of GID. The mean age of the total population was 72.6 years. Patient demographics are presented in Table 1. 
Table 1 Characteristics of the study population included within the retrospective claims database analysis

\begin{tabular}{|c|c|c|c|c|}
\hline Characteristics & All patients, $n(\%)$ & MPR $<0.8, n(\%)$ & $\mathrm{MPR} \geq 0.8, n(\%)$ & $p$ value* \\
\hline Total study population & $15,846(100 \%)$ & $7,244(45.72 \%)$ & $8,602(54.28 \%)$ & - \\
\hline \multicolumn{5}{|l|}{ Age } \\
\hline Age, mean $(\mathrm{SD})$ years & $72.6(11.7)$ & $73.9(11.7)$ & $71.5(11.6)$ & $<0.0001$ \\
\hline $11-20$ & $1(0.01 \%)$ & $0(0 \%)$ & $1(0.01 \%)$ & - \\
\hline $21-30$ & $8(0.05 \%)$ & $5(0.07 \%)$ & $3(0.03 \%)$ & - \\
\hline $31-40$ & $51(0.32 \%)$ & $23(0.32 \%)$ & $28(0.33 \%)$ & - \\
\hline $41-50$ & $437(2.76 \%)$ & $177(2.44 \%)$ & $260(3.02 \%)$ & - \\
\hline $51-60$ & 2,040 (12.87\%) & $784(10.82 \%)$ & $1,256(14.60 \%)$ & - \\
\hline $61-70$ & $3,919(24.73 \%)$ & $1,581(21.82 \%)$ & $2,338(27.18 \%)$ & - \\
\hline $71-80$ & $5,296(33.42 \%)$ & $2,526(34.87 \%)$ & $2,770(32.30 \%)$ & - \\
\hline 81 and older & $4,094(25.84 \%)$ & $2,148(29.65 \%)$ & $1,946(22.62 \%)$ & - \\
\hline \multicolumn{5}{|l|}{ Gender } \\
\hline Male & $9,555(60.30 \%)$ & $4,355(60.12 \%)$ & $5,200(60.45 \%)$ & 0.6701 \\
\hline \multicolumn{5}{|l|}{ Region } \\
\hline East & $4,022(25.38 \%)$ & $1,969(27.18 \%)$ & $2,053(23.87 \%)$ & - \\
\hline Northwest & $5,882(37.12 \%)$ & $2,570(35.48 \%)$ & $3,312(38.50 \%)$ & - \\
\hline South & 4,385 (27.67\%) & $2,083(28.75 \%)$ & $2,302(26.76 \%)$ & - \\
\hline West & $1,557(9.83 \%)$ & $622(8.59 \%)$ & $935(10.87 \%)$ & - \\
\hline \multicolumn{5}{|l|}{ Regimen } \\
\hline Levodopa, but not DA or amantadine & $6,127(38.67 \%)$ & $3,478(48.01 \%)$ & $2,649(30.80 \%)$ & $<0.0001$ \\
\hline DA, but no levodopa or amantadine & $899(5.67 \%)$ & $478(6.60 \%)$ & $421(4.89 \%)$ & $<0.0001$ \\
\hline Levodopa and DA, but not amantadine & $5,389(34.01 \%)$ & $1,974(27.25 \%)$ & $3,415(39.70 \%)$ & $<0.0001$ \\
\hline Amantadine, but not levodopa or DA & $120(0.76 \%)$ & $87(1.20 \%)$ & $33(0.38 \%)$ & $<0.0001$ \\
\hline Amantadine and Levodopa but not DA & $899(5.67 \%)$ & $416(5.74 \%)$ & $483(5.61 \%)$ & 0.7292 \\
\hline Amantadine and DA but not levodopa & $190(1.20 \%)$ & $68(0.94 \%)$ & $122(1.42 \%)$ & 0.0057 \\
\hline Amantadine, DA and levodopa & $2,012(12.70 \%)$ & $613(8.46 \%)$ & $1,399(16.26 \%)$ & $<0.0001$ \\
\hline MAO-B inhibitors (no amantadine, no DA, no levodopa) & $129(0.81 \%)$ & $73(1.01 \%)$ & $56(0.65 \%)$ & 0.0128 \\
\hline $\begin{array}{l}\text { Other PD-related meds (no levodopa, amantadine, } \\
\text { DA, or MAO-B inhibitors) }\end{array}$ & $81(0.51 \%)$ & $57(0.79 \%)$ & $24(0.28 \%)$ & $<0.0001$ \\
\hline \multicolumn{5}{|l|}{ GID diagnosis } \\
\hline GID diagnosis after PD diagnosis & $4,449(28.08 \%)$ & $2,038(28.13 \%)$ & $2,411(28.03 \%)$ & 0.8832 \\
\hline GID diagnosis before PD diagnosis & $1,360(8.58 \%)$ & $653(9.01 \%)$ & $707(8.22 \%)$ & 0.0750 \\
\hline GID diagnosis both before and after PD diagnosis & $4,369(27.57 \%)$ & $2,191(30.25 \%)$ & $2,178(25.32 \%)$ & $<0.0001$ \\
\hline GID diagnosis in-between first and second PD diagnosis & $130(0.82 \%)$ & $67(0.92 \%)$ & $63(0.73 \%)$ & 0.1817 \\
\hline Never diagnosed with GID & $5,538(34.95 \%)$ & $2,295(31.68 \%)$ & $3,243(37.70 \%)$ & $<0.0001$ \\
\hline Ever diagnosed with GID & $10,308(65.05 \%)$ & $4,949(68.32 \%)$ & $5,359(62.30 \%)$ & - \\
\hline \multicolumn{5}{|l|}{ Depression } \\
\hline Depression & $5,495(34.68 \%)$ & $2,649(36.57 \%)$ & $2,846(33.09 \%)$ & $<0.0001$ \\
\hline \multicolumn{5}{|l|}{ Tablets, mean (SD) } \\
\hline Number of PD tablets per day & $3.60(1.86)$ & $3.67(2.09)$ & $3.53(1.63)$ & $<0.0001$ \\
\hline Number of non-PD tablets per day & $9.47(65.31)$ & $9.63(69.27)$ & $9.34(61.78)$ & 0.7847 \\
\hline Number of PD medications & $2.30(1.41)$ & $1.82(1.11)$ & $2.71(1.51)$ & $<0.0001$ \\
\hline Number of PD prescriptions & $12.93(9.78)$ & $7.48(5.12)$ & $17.53(10.39)$ & $<0.0001$ \\
\hline Prescription taken more than once daily & $2,054(12.96 \%)$ & $758(10.46 \%)$ & $1,296(15.07 \%)$ & $<0.0001$ \\
\hline
\end{tabular}

$D A$ dopamine agonist, $G I D$ gastrointestinal disorder, $M A O-B$ monoamine oxidase, $M P R$ medication possession ratio, $P D$ Parkinson's disease, $S D$ standard deviation

* $p$ value obtained from a statistical test comparing patients with MPR $<0.8$ to patients with MPR $\geq 0.8$ (Pearson's chi-square test for categorical variables, two-sample $t$ test for continuous variables) 
3.1 Extent of Non-Compliance in the USA PD Population

Approximately $46 \%$ of patients were found to be noncompliant with their PD-related medication (MPR $<0.8$ ) and the degree of non-compliance was consistent across the four main regions of the USA (Table 1). The mean age of the non-compliant cohort was higher than the mean age of the compliant cohort (74 vs. 72 years; $p<0.0001$ ).

\subsection{Predictors of Non-Compliance}

Results of the regression analysis are presented in Table 2. After analysing the statistical significance of each covariate, gender was removed as a covariate. The reference case was defined as a patient 81 years or older, diagnosed with PD for more than 2 years and who is receiving more than ten tablets per day, where at least one prescription is for a more than once daily tablet. This reference patient has been diagnosed with depression but never diagnosed with GID. The odds of non-compliance in this patient group was estimated at 0.68 .

Age was found to be a predictor of non-compliance, with patients aged 41-80 years at increased odds to be compliant than patients aged 81 years and older $(p<0.0001)$. The treatment regimen itself was also found to influence the odds of non-compliance. Non-compliance was associated with a treatment regimen containing fewer

Table 2 Results from the multivariable logistic regression estimating the probability of non-compliance in patients with Parkinson's disease

\begin{tabular}{|c|c|c|c|c|c|}
\hline Parameter & $\begin{array}{l}\text { Coefficient } \\
\text { estimate }\end{array}$ & $\begin{array}{l}\text { Standard } \\
\text { error }\end{array}$ & $\begin{array}{l}\text { Odds } \\
\text { ratio }\end{array}$ & $\begin{array}{l}\text { Wald } 95 \% \text { confidence } \\
\text { limits }\end{array}$ & $p$ value \\
\hline Reference case & -0.3907 & 0.0562 & 0.68 & $0.61,0.76$ & N/A \\
\hline \multicolumn{6}{|l|}{ Age (years) } \\
\hline $21-30$ & 0.1606 & 0.7451 & 1.17 & $0.27,5.06$ & 0.8294 \\
\hline $31-40$ & -0.2826 & 0.2905 & 0.75 & $0.43,1.33$ & 0.3307 \\
\hline $41-50$ & -0.4777 & 0.1059 & 0.62 & $0.50,0.76$ & $<0.0001$ \\
\hline $51-60$ & -0.5511 & 0.0574 & 0.58 & $0.51,0.65$ & $<0.0001$ \\
\hline $61-70$ & -0.4439 & 0.0467 & 0.64 & $0.59,0.70$ & $<0.0001$ \\
\hline $71-80$ & -0.1343 & 0.0428 & 0.87 & $0.80,0.95$ & 0.0017 \\
\hline 81 and older & Reference $^{\mathrm{a}}$ & & & & \\
\hline \multicolumn{6}{|l|}{ Number of tablets per day } \\
\hline Less than 10 tablets per day & 0.8892 & 0.0341 & 2.43 & $2.28,2.60$ & $<0.0001$ \\
\hline Greater than 10 tablets per day & Reference $^{\mathrm{a}}$ & & & & \\
\hline \multicolumn{6}{|l|}{ Time since diagnosis } \\
\hline Diagnosed for $<2$ years & 0.129 & 0.0341 & 1.14 & $1.06,1.22$ & 0.0002 \\
\hline Diagnosed $>2$ years & Reference $^{\mathrm{a}}$ & & & & \\
\hline \multicolumn{6}{|l|}{ Daily tablets } \\
\hline No prescriptions for a more than once daily tablet & -0.4273 & 0.0504 & 0.65 & $0.59,0.72$ & $<0.0001$ \\
\hline $\begin{array}{l}\text { At least one prescription for a more than once daily } \\
\text { tablet }\end{array}$ & Reference $^{\mathrm{a}}$ & & & & \\
\hline \multicolumn{6}{|l|}{ GID } \\
\hline Had GID & 0.2873 & 0.0361 & 1.33 & $1.24,1.43$ & $<0.0001$ \\
\hline Never had GID & Reference $^{\mathrm{a}}$ & & & & \\
\hline \multicolumn{6}{|l|}{ Depression } \\
\hline No depression diagnosis & -0.2357 & 0.0354 & 0.79 & $0.74,0.85$ & $<0.0001$ \\
\hline Depression diagnosed & Reference $^{\mathrm{a}}$ & & & & \\
\hline
\end{tabular}

Gender proved to be a non-statistically significant covariate during the stepwise selection and was therefore removed from the final model. Odds ratios (on the linear scale) and their confidence intervals were derived from the original coefficient estimates obtained with the logistic regression by exponentiating the values. A higher odds ratio compared to the reference case means that the odds of the patient group being non-compliant is increased (odds of compliance is decreased); a lower value means that the odds of non-compliance in the patient group is decreased compared to the reference case. The 11-20-year age category only included one patient, who was removed from the data prior to running the final model. The goodness of fit was evaluated using the scaled Pearson $\chi^{2}$ test $(15,840.6043, d f=16,000)$

GID gastrointestinal disorder, NA not applicable

${ }^{a}$ Reference selection is arbitrary 
than ten tablets per day $(p<0.0001)$ and at least one prescription for a more than once-daily tablet $(p<0.0001)$. Time since diagnosis also acted as a predictor for noncompliance, with patients diagnosed for $<2$ years at increased odds to be non-compliant than patients diagnosed for a longer period than 2 years $(p=0.0002)$. In terms of comorbidities, those patients with diagnosed with GID $(p<0.0001)$ or depression $(p<0.0001)$ were at increased odds to be non-compliant compared to patients without these diagnoses.

Overall, patients with the lowest odds to be compliant had the following characteristics: aged 81 years or older, prescribed fewer than ten tablets per day but with at least one prescription that required multiple daily doses, had been diagnosed with PD for $<2$ years, and had a diagnosis of both GID and depression.

\subsection{The Healthcare Costs of Patients Who Are Compliant and Non-Compliant}

Overall, the total all-cause annual mean healthcare costs for PD patients were lower for compliant patients $(\$ 77,499)$ than the mean costs for non-compliant patients $(\$ 84,949 ; p<0.0001)$, with compliant patients having annual mean costs that were $\$ 7,451$ higher than noncompliant patients (Table 3). The main drivers of the total mean costs for non-compliant and compliant patients were non-emergency room (inpatient) non-ER hospitalisation mean costs $(24.7 \%$ of the total costs for non-compliant patients vs. $19.4 \%$ of the total costs for compliant patients), and outpatient mean costs (emergency room mean costs, laboratory mean costs, non-laboratory ancillary service mean costs, and physician visit mean costs;
$29.4 \%$ of the total mean costs for non-compliant patients vs. $24.8 \%$ of the total mean costs for compliant patients) (Table 3). Although total drug mean costs were higher for compliant than non-compliant patients $(19.8 \%$ of the total mean costs for non-compliant patients; $30.1 \%$ of the total mean costs for compliant patients) (Table 3 ), driven mainly by the mean costs of PD-related medication (difference of $\$ 5,674$ per year; $p<0.0001$ ), outpatient and non-ER hospitalisation mean costs were both lower for patients compliant to medication (Fig. 3).

\section{Discussion}

Almost half of the patients included within this USA retrospective claims database analysis were considered to be non-compliant with their PD treatment regimen. This is consistent with previous estimates [16] and confirms that non-compliance in PD remains an important healthcare problem. Indeed, non-compliance was associated with total yearly healthcare costs that were $10 \%$ higher than the annual healthcare mean costs for patients compliant with their treatment regimen in the current analysis. Although total drug mean costs are higher for patients who demonstrate better compliance with PD therapy, these mean costs are outweighed by the total out- and inpatient hospitalisation costs. As a result, the total direct all-cause healthcare mean costs are lower for patients compliant with their PD therapy than for patients non-compliant with their prescribed regimen. It should be noted however that the mean costs varied considerably between patients, which is reflected in the large standard deviations observed in this study (Table 3).

Table 3 Healthcare costs (\$US) incurred by PD patients in total and stratified by level of compliance

\begin{tabular}{|c|c|c|c|c|}
\hline Cost type, mean \$US (SD) & All PD patients & $\begin{array}{l}\text { Non-compliant patients } \\
(\mathrm{MPR}<0.8)\end{array}$ & $\begin{array}{l}\text { Compliant patients } \\
(\mathrm{MPR} \geq 0.8)\end{array}$ & $p$ value \\
\hline PD-related medication costs & 7,328.41 (9,648.66) & $4,248.32(6,247.1)$ & $9,922.25(11,132.02)$ & $<0.0001$ \\
\hline Other medication costs & $13,007.62(18,203.82)$ & $12,547.79(16,441.39)$ & $13,394.85(19,557.83)$ & 0.0031 \\
\hline Total drug costs & $20,336.03(21,542.00)$ & $16,796.11(18,261.6)$ & $23,317.1(23,550.5)$ & $<0.0001$ \\
\hline Emergency room visit costs & $15,038.10(30,086.98)$ & $18,348.34(34,037.19)$ & $12,250.46(25,981.15)$ & $<0.0001$ \\
\hline Laboratory investigation costs & $1,153.00(1,931.61)$ & $1,131.11(1,970.29)$ & $1,171.43(1,898.34)$ & 0.1906 \\
\hline Non-laboratory ancillary service costs & $661.54(1,417.82)$ & $680.79(1,449.96)$ & $645.32(1,390.07)$ & 0.1167 \\
\hline Physician visit costs & $4,998.43(5,099.45)$ & $4,852.80(4,954.77)$ & $5,121.06(5,215.32)$ & 0.0010 \\
\hline Outpatient costs & $21,851.07(31,642.94)$ & $25,013.04(35,486.04)$ & $19,188.27(27,724.16)$ & $<0.001$ \\
\hline $\begin{array}{l}\text { Non-emergency room (inpatient) } \\
\text { hospitalisation costs }\end{array}$ & $17,743.77(38,292.28)$ & $20,962.01(41,760.52)$ & $15,033.59(34,879.72)$ & $<0.0001$ \\
\hline Uncategorised costs & $20,973.83(35,680.49)$ & $22,178.19(39,296.31)$ & $19,959.59(32,290.81)$ & $<0.0001$ \\
\hline Total cost & $80,904.69(86,278.16)$ & $84,949.37(92,523.53)$ & $77,498.55(80,492.45)$ & $<0.0001$ \\
\hline
\end{tabular}

$M P R$ medication possession ratio, $P D$ Parkinson's disease, $S D$ standard deviation

$p$ value calculated using Satterthwaite $t$ test method for unequal variances 
Fig. 3 The mean cost difference between outpatient mean costs, drug mean costs, and non-emergency room hospitalisation mean costs for non-compliant patients minus costs for compliant patients undergoing PD treatment. $P D$ Parkinson's disease

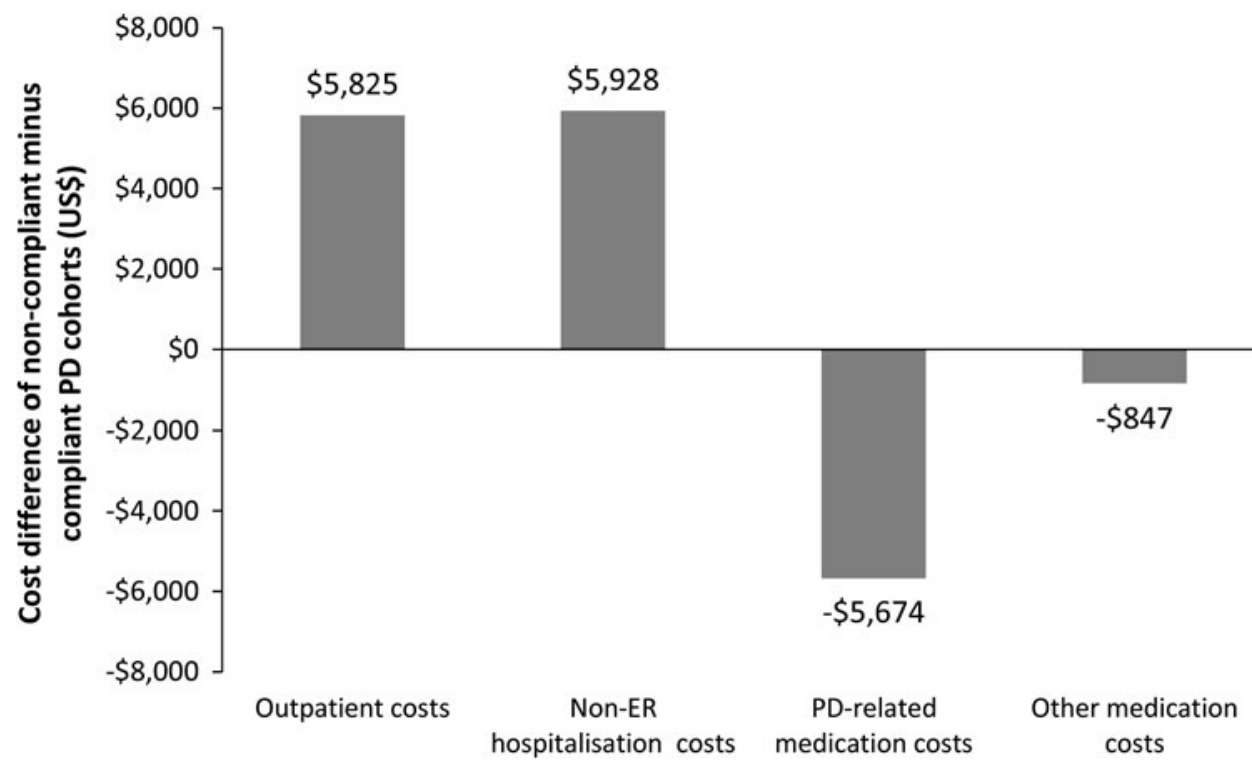

An older retrospective study analysing insurance claims from a small number of health plans between January 1997 and December 2004 also reported that the unadjusted mean medical costs were significantly higher among non-compliant patients than among compliant patients, even though non-compliant patients had lower prescription drug costs than compliant patients [26]. Key drivers of the cost difference between cohorts appear to be outpatient services, hospitalisation, and prescription drugs costs [13]. Thus, patients with PD who are non-compliant with their PDrelated medication may represent a greater burden to the healthcare system than comparable patients who are compliant with their treatment regimen. Further, this current study and previous retrospective analyses have only taken into account the total direct all-cause healthcare costs associated with compliance with PD treatment, not the indirect costs that impact the societal burden of non-compliance in PD (e.g., productivity losses). The indirect medical cost of PD is estimated to be $\$ 16.3$ billion per year in total for the USA (2002 US dollars), and these indirect costs may also be higher for patients who demonstrate poor compliance with PD treatment compared with patients who are compliant [13].

In the current analysis poor compliance was associated with older age ( $>81$ vs. $41-80$ years), time since PD diagnosis of $<2$ years, medication requiring multiple daily doses, and a concomitant diagnosis of depression and/or GID. These identified characteristics were somewhat different to those observed in a recent single-centre observational study of compliance in PD that used electronic monitoring bottles to measure compliance over 3 months [14]. Both studies found that higher depressions scores and medication requiring dosing more frequently than once daily were predictors of non-compliance. However, the previous study found poor compliance was associated with taking more PD-related tablets per day, which is in contrast to the current study, which found that the odds of noncompliance were 2.43 -fold greater in patients taking fewer than ten tablets per day compared with those taking more than ten tablets per day (Table 2). This disparity may be due to differences in the methodology used for the two analyses. In the previous study, patients prescribed more than one PD-related drug had the percentage of the total dose taken calculated separately for each drug and then averaged. For the current study, the "days supplied" from all PD-related drugs prescribed were combined to calculate a single MPR value for each patient rather than calculating an individual MPR for each drug that a patient was prescribed. This approach means that a patient with prescriptions for many different drugs may appear more compliant than a patient with prescriptions for a single drug because of the greater number of "days supplied" present in the claims of those patients.

Importantly, there is expected to be an increase in the number of patients with PD as a result of the increasing average life expectancy of the global population [27]. A study investigating the prevalence of PD in the world's ten most populous countries predicted that the number of individuals over the age of 50 years with PD was between 4.1 million and 4.6 million in 2005 , with this number expected to double to between 8.7 million and 9.3 million by 2030 [5]. If current treatment methods remain the same and the number of patients with PD increases, it may be of interest to improve patient compliance with treatment.

A variety of possible methods of increasing compliance have been developed. These methods include tying the medication-taking process to other daily routines [28], 
development of an individualised treatment plan that simplifies the regimen as far as possible [29], implementation of electronic medication compliance monitoring systems [30], provision of compliance aids such as medication calendars or dispensing systems [29], appropriate patient education [31], providing advanced warning about the possibility of side effects [32], increasing the perceived utility of the medication [33], and decreasing the cost barrier of the medication [34]. Decreasing the complexity of the treatment regimen could also prove to be an important means by which compliance can be improved [35]. For PD specifically, a key method to increase compliance is to improve the ease of administration of the treatment regimen $[36,37]$. Problems with swallowing pills are prevalent in the elderly population, especially in patients with dysphagia [38].

Another strategy for treating PD is the use of a skin patch or transdermal therapeutic system (TTS), which may offer some advantages over oral administration of antiParkinson therapy: patch use could reduce gastrointestinal variations and avoid first-pass metabolism, as well as simplifying the daily dosing schedule and ensuring a short plasma elimination half-life of the drug after patch removal [37]. A transdermal patch that contains the dopamine agonist rotigotine is available in the USA and EU for the treatment of the signs and symptoms of early stage idiopathic $\mathrm{PD}$ and in combination with levodopa for the treatment of late-stage PD [39]. Clinical trials of the transdermal patch demonstrated an average compliance of $98 \%$, with no patient taking $<85 \%$ of the trial medication [40]. The ease of administration offered by the transdermal patch suggests that it may have the potential to reduce healthcare costs associated with non-compliance in patients with PD.

A potential limitation of the current analysis could be the manner in which compliance was measured. The use of claims data necessarily assumes that every prescription filled represents medication consumed by the patient; in reality, the patient may not have visited the doctor to receive a prescription, the prescription may be filled without the patient ingesting the drug, or the prescription may be filled, the drug ingested, but the claim not filed by the patient. Despite this, pharmacy records have been demonstrated to be a valid measure of the exposure of patients to their medication [41] and are recognised as a standard means of assessing compliance in the absence of a more accurate method. Indeed, the impact of any inaccuracy in the method would be to underestimate the extent of non-compliance with therapy and allocate patients as compliant where they are actually non-compliant. It is likely, therefore, that the current analysis is a conservative estimate of the current situation with respect to compliance in PD.
The development of GID has been linked with PDrelated medication [42], and as such diagnosis of a GID comorbidity was considered a likely contributor to noncompliance in patients receiving PD-related medication. However, a second limitation of the current analysis is that some patients classified as diagnosed with GID were actually diagnosed prior to PD diagnosis $(8.6 \%)$ or were diagnosed with GID both before and after PD diagnosis $(27.8 \%)$. Therefore, for patients with GID who were diagnosed prior to PD diagnosis, the GID would not be considered an adverse event associated with PD-related medication and would therefore not be expected to influence compliance. Only GID diagnosed between the first and second PD diagnosis or after the second PD diagnosis (28.9\%) would be expected to influence compliance in this patient population. The inclusion of GID cases diagnosed prior to PD diagnosis may therefore underestimate the impact of PD medication-related GID on the odds of noncompliance with therapy, with the current results considered to be a conservative estimate of the impact of this covariate. It should be noted that a pre-PD diagnosis of GID could be worsened by the administration of PD-related medications and therefore still contribute to non-compliance in this population.

Furthermore, this logistic regression did not account for unobserved factors such as disease severity or socioeconomic background, which may have influenced both compliance and healthcare utilisation. For example, previous studies have recognised a "healthy adherer" effect in which higher compliance with placebo was also associated with improved health outcomes [43]. As the analysis of healthcare costs did not include any regression analysis to control for covariates, any comparisons between the compliant and non-compliant groups must be conducted with caution, especially as all-cause costs were measured rather than just PD-related costs. Simultaneity bias may have been present for any of the covariates that were observed through the follow-up period, during which the MPR was computed. For example, one scenario that could generate simultaneity is if a patient is influenced in their medication compliance by their number of physician or hospital visits.

\section{Conclusions}

Overall, non-compliance with treatment in patients with PD is a significant problem and one that may be associated with an increased economic burden for the healthcare provider. One of the predictors of non-compliance in patients with PD is the necessity for medication administered in multiple daily doses. Development of PD treatments that have an extended release formulation requiring fewer daily doses or have an alternative mode of 
administration could potentially improve compliance. Given the expected increase in the worldwide prevalence of PD in the coming decades, employing strategies to increase compliance may contribute to the reduction of the future burden of PD.

Acknowledgments The authors thank Elizabeth Moorcroft (HERON Evidence Development Ltd.) for her statistical design and analysis, Jim Smyth, Solène Thieffry, and Simon Borghs (UCB Pharma) for their helpful discussions and comments, Ging-Ging Li and Azita Tofighy (UCB Pharma) for their assistance in the preparation of the manuscript, and two anonymous reviewers for their insightful and detailed comments on previous versions of this article. Under direction of the authors, professional medical writing assistance was provided by Ebony Samuels and James Saunders (HERON Evidence Development Ltd.), which was contracted by UCB Pharma. Results from the analysis have been presented at the Academy of Managed Care Pharmacy's Educational Conference, 19-21 October 2011, Atlanta, GA,USA.

Author contributions FFR contributed to the study design, interpretation of data, and critically reviewed the paper. GP contributed to the conception of the paper, supervised the statistical analyses, and critically reviewed the paper. KAM contributed to the study conception and critically reviewed the paper. ES contributed to the interpretation of data and critically reviewed the paper. LEM coordinated and designed the study, analysed and interpreted data, and wrote the paper. LEM will act as guarantor.

Disclosures This study was sponsored by UCB Pharma. FFR is a former employee and KAM, ES and LM are current employees of UCB Pharma. GP (HERON Evidence Development Ltd.) was contracted by UCB Pharma to assist in the analysis of the study.

Open Access This article is distributed under the terms of the Creative Commons Attribution Noncommercial License which permits any noncommercial use, distribution, and reproduction in any medium, provided the original author(s) and the source are credited. The exclusive right to any commercial use of the article is with Springer.

\section{References}

1. de Rijk MC, Launer LJ, Berger K, Breteler MM, Dartigues JF, Baldereschi M, et al. Prevalence of Parkinson's disease in Europe: a collaborative study of population-based cohorts. Neurologic Diseases in the Elderly Research Group. Neurology. 2000;54(11 Suppl 5):S21-3.

2. Canadian Neurological Sciences Federation. Canadian guidelines on Parkinson's disease. Can J Neurol Sci 2012;39(Suppl 4):S1-30.

3. Olanow CW, Stern MB, Sethi K. The scientific and clinical basis for the treatment of Parkinson disease (2009). Neurology. 2009;72(21 Suppl 4):S1-136.

4. Lewitt PA. Levodopa for the treatment of Parkinson's disease. N Engl J Med. 2008;359(23):2468-76.

5. Dorsey ER, Constantinescu R, Thompson JP, Biglan KM, Holloway RG, Kieburtz K, et al. Projected number of people with Parkinson disease in the most populous nations, 2005 through 2030. Neurology. 2007;68(5):384-6.

6. Pinder RM. Drugs for Parkinson's disease: levodopa is still the gold standard. Neuropsychiatr Dis Treat. 2008;4(1):i-ii.
7. Rascol O, Brooks DJ, Korczyn AD, De Deyn PP, Clarke CE, Lang AE. A five-year study of the incidence of dyskinesia in patients with early Parkinson's disease who were treated with ropinirole or levodopa. 056 Study Group. N Engl J Med. 2000;342(20):1484-91.

8. NICE. Parkinson's disease: diagnosis and management in primary and secondary care (Internet). 2011. http://www.nice.org. uk/CG035. Cited 9 Jan 2012.

9. Miyasaki JM, Martin W, Suchowersky O, Weiner W, Lang A. Initiation of treatment of Parkinson's disease (Internet). 2006. http://www.aan.com/professionals/practice/guidelines/pda/ Treatment_PD.pdf. Cited 4 Apr 2012.

10. Schapira AH. Treatment options in the modern management of Parkinson disease. Arch Neurol. 2007;64(8):1083-8.

11. Pahwa R, Factor SA, Lyons KE, Ondo WG, Gronseth G, BronteStewart $\mathrm{H}$, et al. Practice parameter: treatment of Parkinson disease with motor fluctuations and dyskinesia (an evidencebased review): report of the Quality Standards Subcommittee of the American Academy of Neurology. Neurology. 2006;66(7): 983-95.

12. Horstink M, Tolosa E, Bonuccelli U, Deuschl G, Friedman A, Kanovsky $\mathrm{P}$, et al. Review of the therapeutic management of Parkinson's disease. Report of a joint task force of the European Federation of Neurological Societies and the Movement Disorder Society-European Section. Part I: early (uncomplicated) Parkinson's disease. Eur J Neurol. 2006;13(11):1170-85.

13. Huse DM, Schulman K, Orsini L, Castelli-Haley J, Kennedy S, Lenhart G. Burden of illness in Parkinson's disease. Mov Disord. 2005;20(11):1449-54.

14. Grosset KA, Bone I, Grosset DG. Suboptimal medication adherence in Parkinson's disease. Mov Disord. 2005;20(11): $1502-7$.

15. Leopold NA, Polansky M, Hurka MR. Drug adherence in Parkinson's disease. Mov Disord. 2004;19(5):513-7.

16. Tarrants ML, Denarie MF, Castelli-Haley J, Millard J, Zhang D. Drug therapies for Parkinson's disease: a database analysis of patient compliance and persistence. Am J Geriatr Pharmacother. 2010;8(4):374-83.

17. Grosset KA, Bone I, Reid JL, Grosset D. Measuring therapy adherence in Parkinson's disease: a comparison of methods. J Neurol Neurosurg Psychiatry. 2006;77(2):249-51.

18. Hughes DA, Bagust A, Haycox A, Walley T. Accounting for noncompliance in pharmacoeconomic evaluations. Pharmacoeconomics. 2001;19(12):1185-97.

19. Haynes RB, Ackloo E, Sahota N, McDonald HP, Yao X. Interventions for enhancing medication adherence. Cochrane Database Syst Rev. 2008;(2):CD000011.

20. Sackett DL, Haynes RB, Gibson ES, Taylor DW, Roberts RS, Johnson AL. Patient compliance with antihypertensive regimens. Patient Couns Health Educ. 1978;1(1):18-21.

21. Grosset D. Therapy adherence issues in Parkinson's disease. J Neurol Sci. 2010;289(1-2):115-8.

22. Grosset D, Antonini A, Canesi M, Pezzoli G, Lees A, Shaw K, et al. Adherence to antiparkinson medication in a multicenter European study. Mov Disord. 2009;24(6):826-32.

23. O'Brien JA, Ward A, Michels SL, Spyridon T, Brandt N. Economic burden associated with Parkinson disease. Drug Benefit Trends. 2009;21(6):179-90.

24. PharMetrics. PharMetrics database (Internet). 2010. http://www. imshealth.com/portal/site/ims/menuitem.d248e29c86589c9c30e8 1c033208c22a/?vgnextoid $=$ d6952a2e23264310VgnVCM100000 ed152ca2RCRD\&vgnextchannel=c03c90916e442310VgnVCM1 00000ed152ca2RCRD. Cited 16 Aug 2010.

25. Makaroff L, Gunn A, Gervasoni C, Richy F. Gastrointestinal disorders in Parkinson's disease: prevalence and health outcomes in a US claims database. J Parkinsons Dis. 2011;1(1):65-74. 
26. Davis KL, Edin HM, Allen JK. Prevalence and cost of medication nonadherence in Parkinson's disease: evidence from administrative claims data. Mov Disord. 2010;25(4):474-80.

27. Chen JJ. Parkinson's disease: health-related quality of life, economic cost, and implications of early treatment. Am J Manag Care. 2010;16 Suppl Implications:S87-93.

28. Berger BA. Assessing and interviewing patients for meaningful behavioral change: part 1. Case Manag. 2004;15(5):46-50.

29. Bond WS, Hussar DA. Detection methods and strategies for improving medication compliance. Am J Hosp Pharm. 1991; 48(9):1978-88.

30. McKenney JM, Munroe WP, Wright JT Jr. Impact of an electronic medication compliance aid on long-term blood pressure control. J Clin Pharmacol. 1992;32(3):277-83.

31. Ley P, Jain VK, Skilbeck CE. A method for decreasing patients' medication errors. Psychol Med. 1976;6(4):599-601.

32. Galloway R, McGuire J. Determinants of compliance with iron supplementation: supplies, side effects, or psychology? Soc Sci Med. 1994;39(3):381-90.

33. Rohsenow DJ. What place does naltrexone have in the treatment of alcoholism? CNS Drugs. 2004;18(9):547-60.

34. Garfield FB, Caro JJ. Compliance and hypertension. Curr Hypertens Rep. 1999;1(6):502-6.

35. Steinmetz A, Schwartz T, Hehnke U, Kaffarnik H. Multicenter comparison of micronized fenofibrate and simvastatin in patients with primary type IIA or IIB hyperlipoproteinemia. J Cardiovasc Pharmacol. 1996;27(4):563-70.
36. Md S, Haque S, Sahni JK, Baboota S, Ali J. New non-oral drug delivery systems for Parkinson's disease treatment. Expert Opin Drug Deliv. 2011;8(3):359-74.

37. Di Stefano A, Sozio P, Iannitelli A, Cerasa LS. New drug delivery strategies for improved Parkinson's disease therapy. Expert Opin Drug Deliv. 2009;6(4):389-404.

38. Llorca P-M. Discussion of prevalence and management of discomfort when swallowing pills: orodispersible tablets expand treatment options in patients with depression. Ther Deliv. 2011; 2(5):611-22.

39. Sanford M, Scott LJ. Spotlight on rotigotine transdermal patch in Parkinson's disease. Drugs Aging. 2011;28(12):1015-7.

40. Schnitzler A, Leffers KW, Hack HJ. High compliance with rotigotine transdermal patch in the treatment of idiopathic Parkinson's disease. Parkinsonism Relat Disord. 2010;16(8):513-6.

41. Lau HS, de Boer A, Beuning KS, Porsius A. Validation of pharmacy records in drug exposure assessment. J Clin Epidemiol. 1997;50(5):619-25.

42. Salat-Foix D, Suchowersky O. The management of gastrointestinal symptoms in Parkinson's disease. Expert Rev Neurother. 2012;12(2):239-48.

43. Simpson SH, Eurich DT, Majumdar SR, Padwal RS, Tsuyuki RT, Varney J, et al. A meta-analysis of the association between adherence to drug therapy and mortality. BMJ. 2006;333:15-20. 